\title{
Inhibition of return for faces
}

\author{
TRACY L. TAYLOR \\ Dalhousie University, Halifax, Nova Scotia, Canada \\ and \\ MEGAN E. THERRIEN \\ University of Ottawa, Ottawa, Ontario, Canada
}

\begin{abstract}
Inhibition of return (IOR) refers to slower reaction times when a target appears unpredictably in the same location as a preceding cue, rather than in a different location. In the present study, frontal images of human faces were presented intact as face configurations, were rearranged to produce scrambled-face configurations, or were pixilated and randomized to produce nonface configurations. In an orienting paradigm designed to elicit IOR, face and scrambled-face stimuli were used as cues (Experiment 1), as targets (Experiment 2), or along with pixilated nonface stimuli as both cues and targets (Experiment 3). The magnitude of IOR for subsequent localization targets was unaffected by cue configuration. Likewise, the magnitude of IOR was unaffected by target configuration. These results suggest that IOR is a "blind" mechanism that is unaffected by the mere occurrence of biologically relevant cue and target stimuli.
\end{abstract}

Inhibition of return (IOR) refers to slower reaction times (RTs) when a target appears in the same location as a preceding onset cue, rather than in a different location (Posner \& Cohen, 1984). This is true when the visual onset cue occurs at least $300 \mathrm{msec}$ in advance of the target and when its location is nonpredictive of target location. IOR has been explained as an inhibitory aftereffect of attentional withdrawal from the cued location (e.g., Danziger \& Kingstone, 1999). The onset cue captures attention automatically, but because there is no incentive to maintain attention at the cued location (i.e., because the cue location does not predict the target location), attention is withdrawn. A hypothetical location-based inhibitory tag marks the location as visited, and the result is slowed reorienting of attention to targets that appear subsequently in the same location (see Posner, Rafal, Choate, \& Vaughan, $1985)$ and/or a bias against making responses to targets that appear subsequently in the tagged location (see Klein \& Taylor, 1994; Taylor \& Klein, 1998, 2000). Regardless of the mechanism involved, it is believed that IOR subserves the evolutionary function of promoting novelty in visual search by preventing repeated sampling of the same

This work was supported by funding from NSERC to T.L.T. We thank Jennifer Stoltz and two anonymous reviewers for their thoughtful comments on an earlier version of this manuscript. We also thank our participants for volunteering for this study, Kyle Owens for piloting Experiment 2, Alison Moss for collecting data in Experiment 3, Jeff Hancock for providing the human face stimuli created by Jack Loney (jack@ groovinhouse.com), and Carl Helmick for creating the pixilated nonface stimuli used in Experiment 3. Correspondence concerning this article should be addressed to T. L. Taylor, Department of Psychology, Life Sciences Centre, Dalhousie University, Halifax, NS, B3H 4JI Canada (e-mail: ttaylor2@dal.ca). locations (see Klein, 1988; Klein \& MacInnes, 1999). IOR may also have the function of slowing responding enough that behavior can be altered online in response to sudden changes in the environment (see Ivanoff \& Taylor, in press).

The suggestion that IOR may have evolved to allow for adaptive search and responding raises the question of whether the biological significance of visual events affects the magnitude or occurrence of the inhibition. The purpose of the present study was to address this question. Under the assumption that human evolution has depended critically on the processing of social stimuli-particularly, human faces - the goal was to determine how these stimuli affect IOR under natural viewing conditions (i.e., when the eyes are free to move). The interest was not in whether the social context of face stimuli influences the magnitude of IOR but in whether the occurrence of social stimuli per se would have an effect. There is no question that social context can influence attentional orienting and IOR. For example, nonpredictive gaze cues initiate rapid reflexive shifts of visual attention (e.g., Friesen \& Kingstone, 1998; Hietanen \& Leppänen, 2003), emotional (angry and happy) cues slow the disengagement of attention (e.g., Fox, Russo, \& Dutton, 2002), and threat (angry) cues are particularly effective at attracting attention (e.g., Eastwood, Smilek, \& Merikle, 2001; Öhman, Lundqvist, \& Esteves, 2001) but - perhaps because of slow disengagement - are relatively poor at inducing IOR (Fox et al., 2002). Rather than asking about whether the social context communicated by face stimuli (e.g., their direction of gaze or emotional valence) influences IOR, the key question was about whether the presumed biological significance of intact face configurations per se would affect the magnitude of IOR. 
In Experiment 1, human face stimuli were presented as cues in a paradigm designed to elicit IOR. The expectation was that evolution might very well have predisposed us to differentially attend to biologically relevant stimuli. More particularly, it seemed likely that attention would be differentially attracted to and/or maintained on intact face cues versus otherwise identical scrambled-face cues, despite neutral emotional valence. Given that IOR is masked by the dwelling of attention at the cued location (see Danziger \& Kingstone, 1999), this would be expected to result in smaller magnitude IOR effects following face than following scrambled-face cues. To anticipate the results, this prediction was not borne out.

Even if face and scrambled-face cues produce IOR effects that are of equivalent magnitudes, it is possible that such stimuli are differentially sensitive to the effects of IOR. Although this prediction may seem strange in light of the apparent proactive influence of IOR on target RTs, it is nevertheless possible, depending on the locus of the IOR effects. Consider that, under natural viewing conditions (i.e., when the eyes are free to move), IOR seems to reflect operations working at a relatively late stage in the information-processing stream (Kingstone \& Pratt, 1999; Taylor \& Klein, 2000). More particularly, when the eyes are free to move (as in the present experiment), IOR appears to reflect a bias against responding to targets that arise at the cued location (see Klein \& Taylor, 1994; see also Taylor \& Klein, 1998). As such, any processing differences associated with face stimuli, relative to scrambled-face ones, could contribute to differential accumulation of evidence during this delay, effectively reducing the impact of IOR on face targets, relative to scrambled-face ones. Experiment 2 tested this possibility by presenting intact face and scrambled-face configurations as targets. The configuration of the target was task irrelevant; participants made speeded responses to localize the target to the left or the right. The expectation was that even when task irrelevant, face configurations would escape the effects of IOR more readily than would scrambled-face configurations that otherwise shared the same visual features (i.e., that face target configurations would reveal a relatively smaller magnitude IOR effect). The results of Experiment 2 suggested that this might be the case, but this was disconfirmed in Experiment 3.

In Experiment 3, intact face, scrambled-face, and pixilated nonface stimuli were presented as both cues and targets. The results revealed no differences in IOR as a function of either cue or target configuration.

\section{EXPERIMENT 1}

In Experiment 1, we examined the effects of spatially nonpredictive face and scrambled-face cues on the magnitude of IOR. IOR was calculated as the difference in RTs between localizing onset targets to the cued location and localizing them to the uncued location. Under the assumption that intact face stimuli might be particularly likely to attract and retain attentional resources, the expectation was that IOR would be fully or partially masked by atten- tional facilitation (see Danziger \& Kingstone, 1999) following face cues, but not following scrambled-face cues. The net result would be a smaller magnitude IOR effect following face cues than following scrambled-face cues.

\section{Method}

Participants. Forty-one Dalhousie undergraduate volunteers participated in exchange for psychology course credit. The participants were tested individually in a dimly illuminated room in a single session that lasted no more than $1 \mathrm{~h}$. All were naive as to the purpose of the experiment.

Stimuli and Apparatus. Three black 2-point outline boxes were presented in a uniform white field on a ViewSonic PT775 17-in. color display monitor. The middle (fixation) box was centered on the computer monitor. At a viewing distance of approximately $56 \mathrm{~cm}$, each box was $2^{\circ}$ of visual angle on a side, with $2^{\circ}$ separation between adjacent edges.

The fixation stimulus consisted of crosshairs, subtending $0.5^{\circ}$ of visual angle, that were centered in the middle box. The peripheral cue stimulus used to establish IOR was a high-pass filtered image of a human face that revealed essential features of the eyes, nose, and mouth. This image was presented intact as a face configuration or else was rearranged to produce a scrambled-face configuration (as can be seen in Figure 1). The cue to fixation was a 2-point black outline circle that filled the middle stimulus box. The target was an identical outline circle that filled the left or the right stimulus box.

The presentation of the stimuli and the collection of data were controlled by a Macintosh G4-400 computer running PsyScope Version 5.1.2 (Cohen, MacWhinney, Flatt, \& Provost, 1993). The participants responded using a Macintosh G4 universal serial bus keyboard.

Procedure. As is shown in Figure 1, a trial began with an 800 -msec presentation of the fixation stimulus; this stimulus remained visible throughout the trial, except during the presentation of the fixation cue (see below). This was followed by a $200-\mathrm{msec}$ presentation of the cue. The cue was equally likely to be a face or a scrambled-face configuration and was equally likely to appear in the left or the right stimulus box. The scrambled-face cues were visually identical to the face cues, except for whether the features were organized into a holistic face representation or not; these cues were intended to provide a baseline measure against which IOR following face cues could be compared. The intention of the decision to mix face and scrambled-face cues within blocks was to reduce the possibility of the participants' adopting a different cognitive set for each type of cue. After a 300-msec delay, the fixation cue was presented for $200 \mathrm{msec}$. The participants were instructed not to respond to any of these events. If the participants failed to comply with this instruction, they were given an error message, and the trial was aborted and recycled randomly in the trial block. Otherwise, following a further 300 -msec delay, a target appeared for $200 \mathrm{msec}$ with equal probability in the left or the right stimulus box.

The participants were informed that the cues would be faces or scrambled faces but that neither their configuration nor their location would be predictive of target location. The participants were instructed to localize the targets as quickly and as accurately as possible. They responded by using the index finger of the left hand to depress the "z" key of the computer keyboard if the target appeared to the left and the index finger of the right hand to depress the " $m$ " key if the target appeared to the right. Distinct auditory feedback occurred following correct responses made within $1,000 \mathrm{msec}$ of target presentation and following responses made by pressing the incorrect key. Failures to respond within 1,000 msec of target onset were associated with auditory feedback, as well as with a visual message that reported the lack of a valid response and that instructed the participants to depress the space bar to continue; such trials were not recycled.

Because any evolutionary mechanism would undoubtedly have evolved to operate under normal viewing conditions and because 


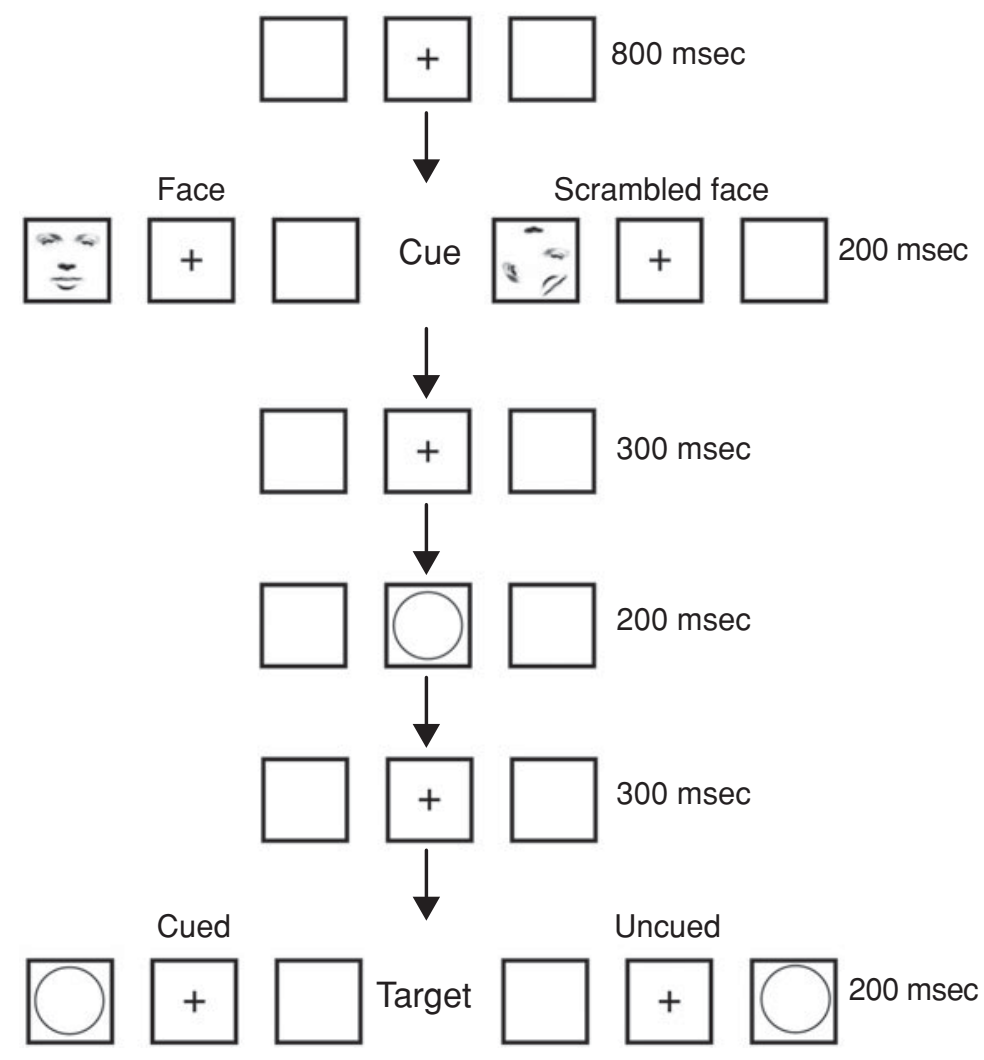

Figure 1. Schematic of the method used in Experiment 1. The cue is shown to the left but, in the experiment, occurred equally often to the right and the left. Note that the distance of the stimulus boxes from one another is not shown to scale. See the text for further details.

the nature of IOR appears to be altered by the explicit monitoring of stable eye position (e.g., Taylor \& Klein, 2000), no attempt was made to constrain or prevent eye movements.

Practice trials were drawn randomly from the experimental block and were presented until the participants reported feeling comfortable with the task. Practice data were neither saved nor analyzed. The experimental block consisted of 320 trials that comprised 40 repetitions of the factorial combination of cue configuration (face or scrambled face), cue location (left or right), and target location (left or right). For the purpose of analysis, the factors of cue and target location were collapsed according to whether the target occurred in the cued (left-left or right-right) or the uncued (left-right or right-left) location. ${ }^{1}$ IOR was revealed as an effect of target location and was calculated as the difference of cued minus uncued RTs; positive values reflect IOR.

\section{Results}

Only trials on which a correct response was executed within 100-1,000 msec of target onset were included in the analysis of RTs. These mean RTs are shown in Figure 2. As well, in Figure 2, the overall accuracies for each condition are displayed; these percent correct scores represent the percentage of trials in each condition that contributed to the calculation of the corresponding mean RT.

An ANOVA on the RT data revealed only a significant main effect of target location $\left[F(1,40)=9.16, M S_{\mathrm{e}}=\right.$ $458.65, p<.01]$, indicative of an overall IOR effect. The effect of cue configuration (face or scrambled face) was not significant $(F<1)$. Likewise, the interaction of cue configuration with target location was not significant $(F<1)$. This lack of significance is not surprising, given that a numerically identical $10-\mathrm{msec}$ IOR effect occurred following face cues $\left[F(1,40)=35.98, M S_{\mathrm{e}}=62.66, p<\right.$ $.01]$ and scrambled-face cues $\left[F(1,40)=31.16, M S_{\mathrm{e}}=\right.$ $62.66, p<.01]$. A sign test for the difference in magnitude of IOR following face and scrambled face cues was not significant [scrambled-face IOR - face IOR: $(n+)=$ $20,(n-)=21, p=1]$.

An equivalent analysis of the accuracy data (see Figure 2) revealed no significant effects (all $p \mathrm{~s}>.22$ ).

\section{Discussion}

The results of Experiment 1 clearly demonstrate that face and scrambled-face cues generate equivalent IOR effects for a subsequent localization target. These findings are consistent with those of Fox et al. (2002, Experiment 3). In a study that explored the effects of emotional valence on IOR, Fox et al. presented high- and low-anxious individuals with face and scrambled face cues, followed at a stimulus onset asynchrony (SOA) of $960 \mathrm{msec}$ (as compared with our 1,000-msec SOA) by a target requiring a speeded localization. Unlike the present study, which 


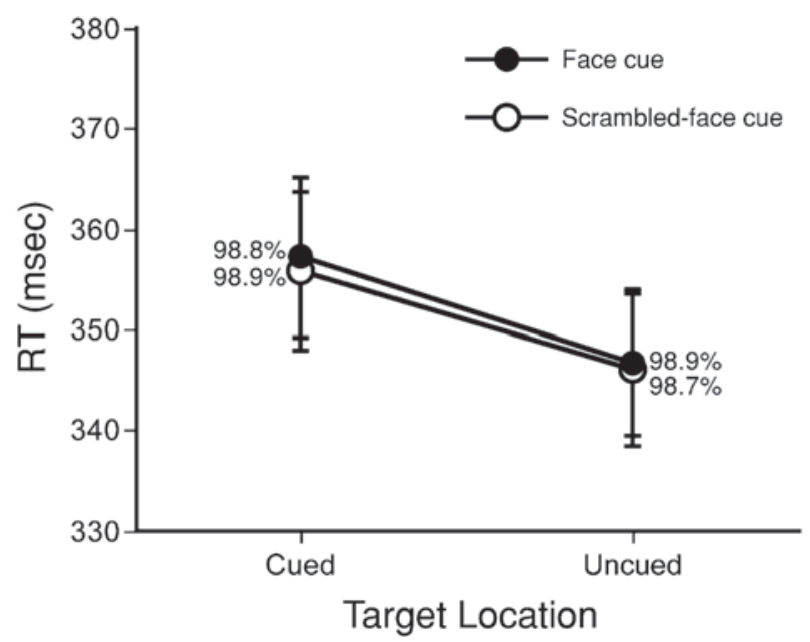

Figure 2. Experiment 1: target reaction times (RTs) as a function of target location (cued or uncued) and cue configuration (face or scrambled face). Error bars show the standard error of the mean. The corresponding percentage of trials (i.e., the percent correct) contributing to each mean is indicated beside the relevant plot.

used images of real faces, the cues in Fox et al.'s study were all simple schematic line drawings. Their face cues exhibited angry or neutral expressions; their scrambled faces consisted of rearranged features of the angry faces, bounded by the outer circle of the schematic drawing. Because their neutral face and scrambled-face cues were most similar to our own face and scrambled-face cues, this is the comparison upon which we will focus (i.e., we will ignore their angry face condition). Moreover, because their stimuli produced differential results with their high- and low-anxious individuals and an adjunct mood induction procedure produced pre-post differences in the high-anxious, but not in the low-anxious, group, we will focus on the results of the latter. This group of low-anxious, non-mood-induced participants, to whom our own participants were probably most similar, revealed IOR effects of $13 \mathrm{msec}$ following neutral face cues and a near-identical 12-msec effect following scrambled-face cues. These effects not only were statistically equivalent to one another, but also were numerically very similar to the 10-msec IOR effects that we observed following our face and scrambled-face cues.

Even though committing a Type II error is always a concern when the null hypothesis is supported, we feel confident in the reliability of our Experiment 1 results. Our confidence derives from several sources. First, whereas the majority of IOR studies have utilized samples of 1220 participants, our sample size was more than twice as large, at 41. Second, the magnitude of the IOR effects that we observed following face and scrambled-face cues were numerically identical and statistically indistinguishable; there was no hint of any difference between these cue conditions. Third, our results closely replicate the numeri- cal and statistical pattern of results obtained in similar conditions in Fox et al.'s (2002) study. Thus, we believe that our data provide support for the view that IOR is a consequence of a spatial nonpredictive visual onset but that its generation is insensitive to the potential biological significance of that onset. This finding was contrary to what we predicted and will be considered further in the General Discussion section.

\section{EXPERIMENT 2}

Even if face and scrambled-face cues generate equivalent IOR effects, the question remains as to whether RTs to face and scrambled-face targets are equally sensitive to its effects. To address this question, Experiment 2 reversed the cue and target assignments in Experiment 1, such that the peripheral onset cues consisted of featureless circles and the targets consisted of face and scrambledface stimuli. The expectation was that face targets may be relatively immune to the effects of IOR that are generated by the peripheral onset cues. This could be argued for on the grounds that human face processing is "special" and that there would be an evolutionary advantage to allowing such stimuli to escape from the effects of inhibitory spatial tagging. More tangibly, it could also be argued for on the grounds that selective processing of face stimuli seems to occur in areas of the ventral visual-processing stream (e.g., the fusiform face area; see Kanwisher, McDermott, \& Chun, 1997; Tong, Nakayama, Moscovitch, Weinrib, \& Kanwisher, 2000), whereas IOR seems to depend on structures associated with the dorsal stream - particularly, the superior colliculus (e.g., Posner et al., 1985; Sapir, Soroker, Berger, \& Henik, 1999) or areas upstream of this structure (Dorris, Klein, Everling, \& Munoz, 2002). Assuming that responses occur after the binding of object features and location, reliance on two different processing streams would provide a mechanism for human face processing to escape the deleterious effects of inhibitory spatial tagging.

Regardless of the neural mechanism that might allow face targets to overcome or escape the effects of IOR, our prediction was that the magnitude of IOR would be relatively smaller for face targets than for scrambled-face targets.

\section{Method}

Participants. Twenty-eight Dalhousie University undergraduate students participated in exchange for psychology class credit. They were naive as to the experimental purpose, and all reported normal or corrected-to-normal vision. None had participated in Experiment 1 .

Stimuli and Apparatus. The stimuli and apparatus were identical to those in Experiment 1, except that the stimuli used as cues and targets were interchanged.

Procedure. The procedure was identical to that in Experiment 1, apart from the stimuli used as cues and targets. The trial events are depicted in Figure 3. As is shown, in Experiment 2, the peripheral cue used to establish IOR was a featureless circle requiring no response; the target was the equiprobable presentation of a face or a scrambled face to the left or right of center. Regardless of its configuration, the participants responded to the target by making a speeded localization response. 


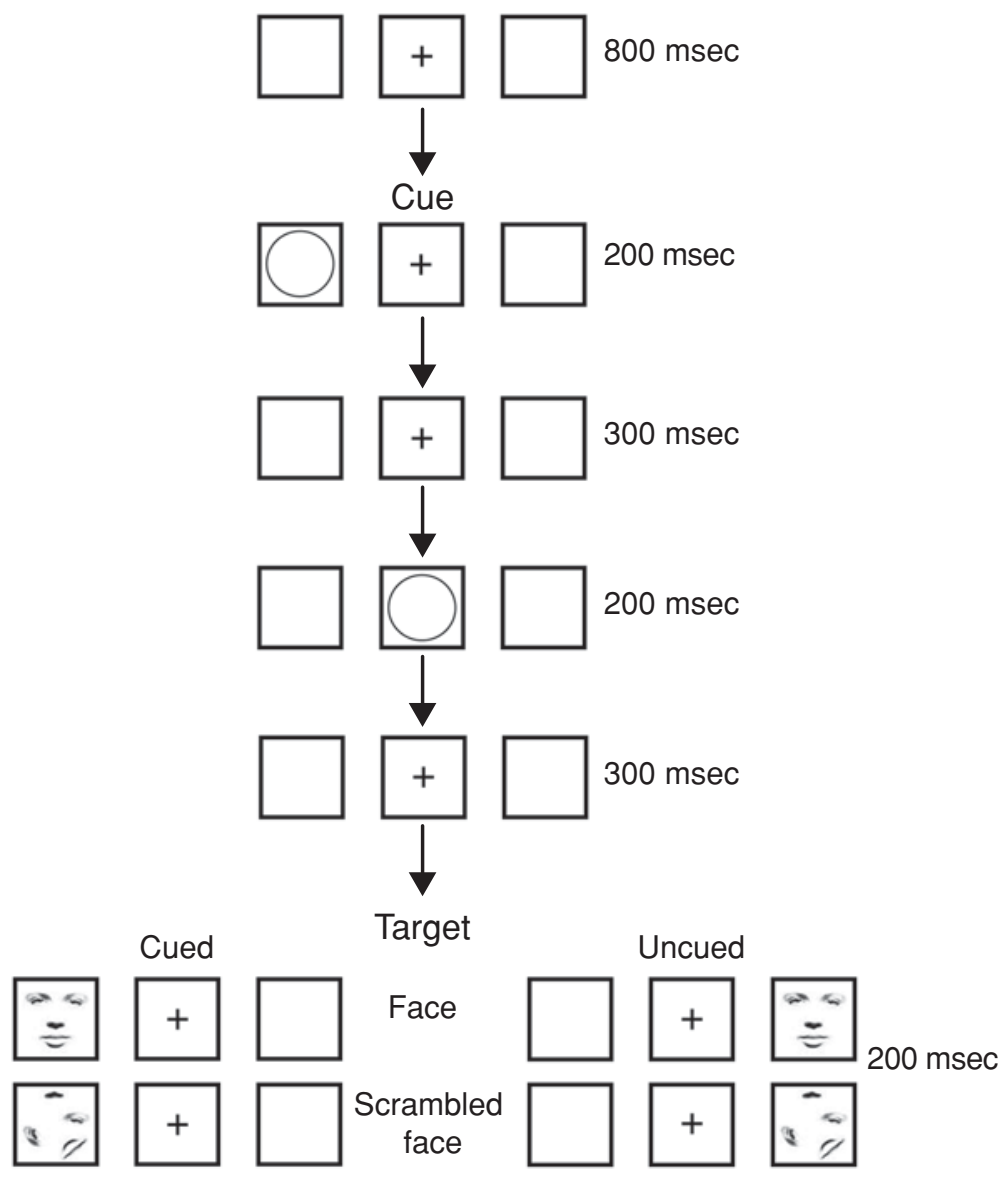

Figure 3. Schematic of the method used in Experiment 2. The cue is shown to the left but, in the experiment, occurred equally often to the right and the left. Note that the distance of the stimulus boxes from one another is not shown to scale. See the text for further details.

\section{Results}

Mean RTs were calculated for only those trials on which the participants responded correctly within 100 $1,000 \mathrm{msec}$ of target presentation. These RT data are shown in Figure 4, along with the associated accuracies.

A two-way ANOVA was performed on the RT data. This analysis revealed a significant main effect of target location $\left[F(1,27)=8.16, M S_{\mathrm{e}}=391.44, p<.01\right]$, consistent with an overall IOR effect. There was no overall effect of target configuration $(F<1)$. There was, however, an interaction of target configuration with target location $\left[F(1,27)=4.70, M S_{\mathrm{e}}=67.00, p<.04\right]$. This interaction was in the predicted direction. In particular, this interaction indicated that IOR was significantly smaller for face targets $[M=7 \mathrm{msec} ; F(1,27)=11.224, p<.01]$ than for scrambled-face targets $[M=14 \mathrm{msec} ; F(1,27)=41.167$, $p<.01]$. Importantly, however, this pattern of results was not found in a significant number of the participants. This was demonstrated by a nonsignificant sign test of the difference in IOR magnitude for face and scrambled-face targets [scrambled-face IOR - face IOR: $(n+)=17$, $(n-)=11, p=.27]$.
An equivalent two-way analysis of accuracy (see Figure 4) revealed no significant effects (all $p \mathrm{~s}>.18$ ).

\section{Discussion}

The difference in magnitude of IOR for face and scrambled-face targets is consistent with the view that face stimuli might be particularly salient to human observers and that processing of these stimuli is thought to rely on a different visual pathway (ventral) from the one thought to represent IOR (dorsal). Even so, a sign test demonstrated that this magnitude difference was not revealed by a significant number of the participants. This suggests caution in interpreting this difference.

We reasoned that even if the magnitude difference revealed in this experiment turns out to be unreliable (as is suggested by the nonsignificant sign test), it is nevertheless still possible that the magnitude of IOR is affected by the presence of face stimuli. Consider the visual characteristics of the scrambled-face stimulus. We conceived of this stimulus as a useful control for the face stimulus by virtue of the fact that it was identical in every aspect, except the organization of the features into a holistic face 


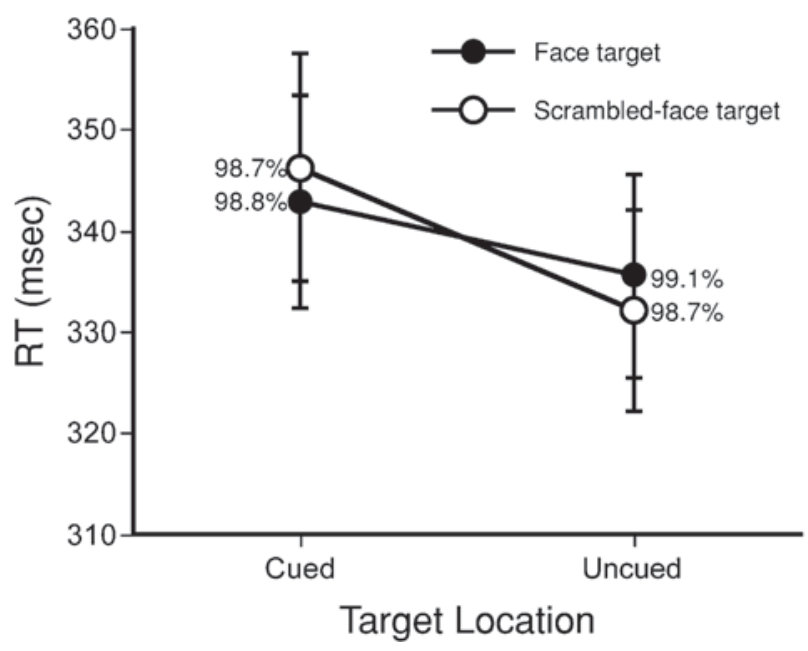

Figure 4. Experiment 2: target reaction times (RTs) as a function of target location (cued or uncued) and target configuration (face or scrambled face). Error bars depict the standard error of the mean. The corresponding percentage of trials (i.e., the percent correct) contributing to each mean is indicated beside the relevant plot.

representation. Nevertheless, facial features were clearly discernible in the scrambled-face stimulus and may have been salient, especially given the mixed-blocks design in which the same features were sometimes arranged as intact faces. To the extent that face processing might be engendered by the mere presence of facial features (especially eyes; see Gallup, Nash, \& Ellison, 1971), regardless of their configuration, the face and scrambled-face stimuli might not have been perceived and processed as differently as we had hoped. If this were true, the failure to find any difference in the magnitude of IOR for face and scrambledface cues in Experiment 1 might reflect the fact that both cues were processed as faces. Likewise, the apparent difference in the magnitude of IOR for face and scrambledface targets in Experiment 2 might have underestimated any advantages that actually existed for the face targets. In Experiment 3, we addressed these possibilities.

\section{EXPERIMENT 3}

Experiment 3 included a nonface stimulus that consisted of a random rearrangement of the pixels contained within the face (and by implication, the scrambled-face) image. This pixilated nonface stimulus was, therefore, matched for overall luminance with the face and scrambled-face stimuli but contained no discernible facial features. All three stimuli were incorporated into a completely mixed factorial design in which cues and targets could be faces, scrambled faces, or nonfaces.

By including a pixilated nonface stimulus in Experiment 3, we were able to determine whether Experiments 1 and 2 underestimated any effects of face stimuli that might otherwise have been observed. To this end, we were particularly interested in whether face, scrambled-face, and nonface cues would produce different magnitude IOR effects and in whether face, scrambled-face, and nonface targets would reveal different magnitude IOR effects. By mixing all three cue and target configurations, we also allowed for the possibility of examining any cue-target interactions that might arise.

\section{Method}

Participants. Twenty-nine Dalhousie University undergraduate students participated in exchange for psychology class credit. They were naive as to the experimental purpose, and all reported normal or corrected-to-normal vision. None had participated in Experiment 1 or 2

Stimuli and Apparatus. In Experiment 3, three stimuli were employed as cues and targets. Two of these stimuli were the face and scrambled-face stimuli from Experiments 1 and 2. However, a third stimulus was created by selecting the pixels from the face stimulus and rearranging them randomly within an imaginary square that bounded the same vertical and horizontal extent as that which contained the face stimulus. This pixilated nonface stimulus is shown in Figure 5 (inset). Otherwise, the stimuli and apparatus were identical to those in Experiments 1 and 2.

Procedure. The design was a $2 \times 3 \times 2 \times 3$ factorial that included cue location (left or right), cue configuration (face, scrambled face, or nonface), target location (left or right), and target configuration (face, scrambled face, or nonface). There were a total of 360 trials, with equal numbers of trials randomly presented from each cell of the design. For the purpose of analysis, the factors of cue and target location were collapsed according to whether the target occurred in the cued (left-left or right-right) or the uncued (left-right or right-left) location. In all other respects, the procedure was identical to that in Experiments 1 and 2.

\section{Results}

Mean RTs were calculated for only those trials on which the participants responded correctly within 100 $1,000 \mathrm{msec}$ of target presentation. These RT data are shown in Figure 5, along with the associated accuracies.

A three-way ANOVA was performed on the RT data, with target location (cued or uncued), cue configuration (face, scrambled face, or nonface), and target configuration (face, scrambled face, or nonface) as factors. This analysis revealed only two significant effects. One was the main effect of target location $[F(1,28)=$ $\left.12.15, M S_{\mathrm{e}}=1,331.94, p<.01\right]$, indicating an overall IOR effect. The other was the main effect of target configuration, with overall longer RTs to the nonface target $(M=338 \mathrm{msec})$ than to the face $(M=329 \mathrm{msec})$ and scrambled-face $(M=330 \mathrm{msec})$ targets. This difference is consistent with overall shorter target RTs in Experiment 2 (face and scrambled-face targets) than in Experiment 1 (open circle targets) and suggests that (1) the face and scrambled-face stimuli may have been particularly salient and (2) the pixilated nonface stimuli were, indeed, functionally equivalent to the open circle stimuli used in Experiments 1 and 2.

As in Experiment 1, cue configuration did not produce any significant difference in the overall RTs $[F(2,56)=$ $\left.2.15, M S_{\mathrm{e}}=380.30, p>.12\right]$ or magnitude of IOR $(F<$ 1). The magnitude of IOR was 9 msec following face cues $[F(1,56)=8.49, p<.01], 13 \mathrm{msec}$ following scrambled- 

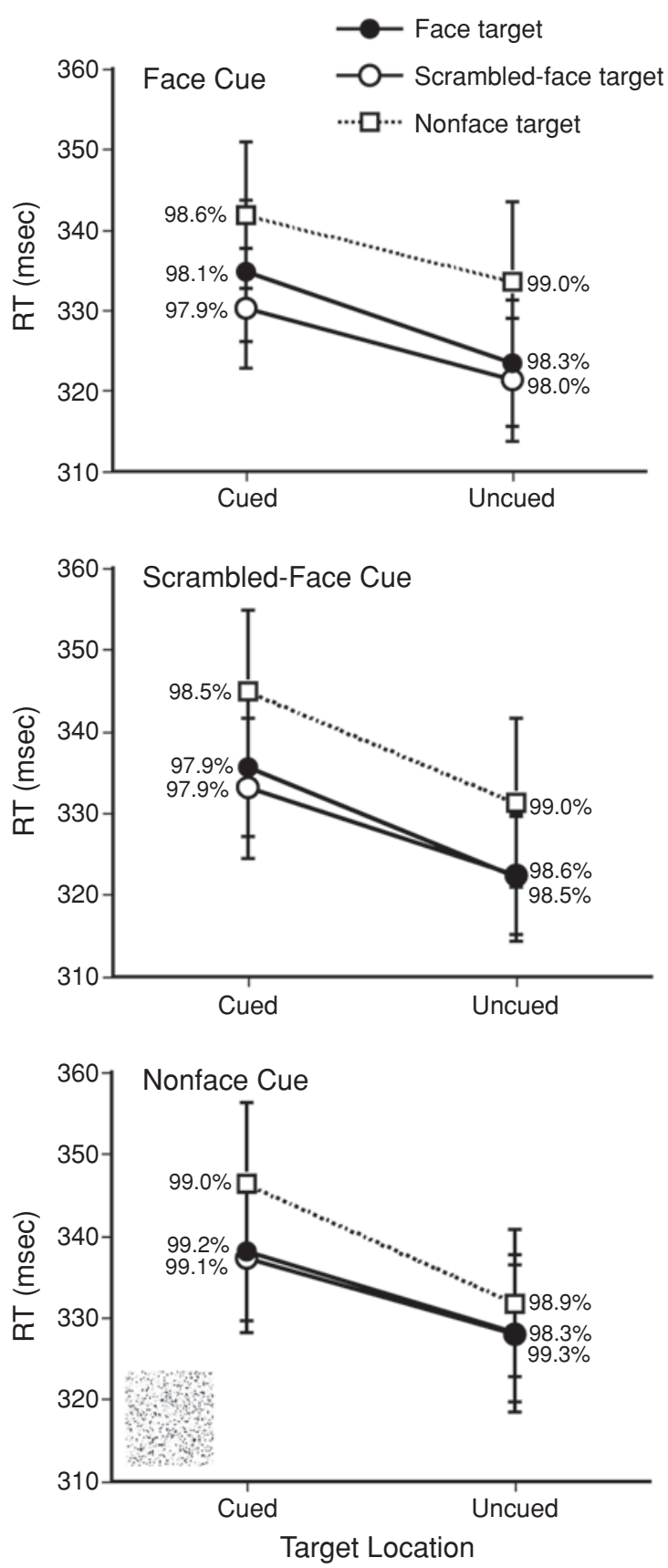

Figure 5. Experiment 3: target reaction times (RTs) as a function of target location (cued or uncued), cue configuration (face, scrambled face, or nonface), and target configuration (face, scrambled face, or nonface). Error bars depict the standard error of the mean. The corresponding percentage of trials (i.e., the percent correct) contributing to each mean is indicated beside the relevant plot. The pixilated nonface stimulus used as both cue and target is inset in the bottom left panel, which depicts the nonface cue data.

face cues $[F(1,56)=15.01, p<.01]$, and $11 \mathrm{msec}$ following nonface cues $[F(1,56)=11.98, p<.01]$.

The results failed to replicate or extend those of Experiment 2 , since there was no significant difference in the magnitude of the IOR effect as a function of target configuration $(F<1)$. IOR was a near-identical $11 \mathrm{msec}$ for face targets $[F(1,56)=12.78, p<.01], 12 \mathrm{msec}$ for scrambled-face targets $[F(1,56)=8.81, p<.01]$, and $10 \mathrm{msec}$ for nonface targets $[F(1,56)=14.09, p<.01]$. A sign test revealed no significant difference in the number of the participants who showed larger versus smaller IOR effects for face versus nonface targets [nonface IORface IOR: $(n+)=15,(n-)=14, p=1]$.

There was also no significant interaction between cue configuration and target configuration $(F<1)$ and no three-way interaction of these factors with target location $(F<1)$.

An equivalent ANOVA of percent correct scores revealed only a small but significant main effect of cue configuration $\left[F(2,56)=3.89, M S_{\mathrm{e}}=5.82, p<.03\right]$. Responses were correct on an average of $98 \%$ of the trials with a face cue, $98 \%$ of the trials with a scrambled-face cue, and $99 \%$ of the trials with a nonface cue. No other effects were significant (all $p$ s $>.32$ ).

\section{Discussion}

Experiment 3 revealed no differences in the magnitude of IOR as a function of cue and/or target configuration. Whether cues were intact faces, scrambled faces, or pixilated nonfaces, IOR was of equivalent magnitude. The equivalence of IOR following face and scrambled-face cues replicates the results of Experiment 1, and the equivalence of IOR following all three types of cue demonstrates that underestimation due to a comparison of face cues against scrambled-face cues was not an issue.

As with the cue stimuli, whether targets were configured as intact faces, scrambled faces, or pixilated nonfaces, IOR was of equivalent magnitude. The fact that IOR was equivalent for face and scrambled-face targets represents a nonreplication of the apparent difference shown in Experiment 2; however, in light of the nonsignificant Experiment 2 sign test, this failure to replicate is not surprising. Importantly, the fact that there was also no difference in IOR for face and nonface targets in Experiment 3 demonstrates that, as was true for Experiment 1, underestimation due to a comparison of face targets against scrambledface targets was not an issue in Experiment 2.

\section{GENERAL DISCUSSION}

The results of this study are clear. An onset cue, whatever its stimulus configuration, is capable of generating an inhibitory effect that influences the speed with which a subsequent target stimulus is localized, whatever its (the target's) configuration.

At first blush, the finding that cue configuration does not affect the magnitude of IOR seems surprising in light of the fact that Tipper, Grison, and Kessler (2003) have shown a long-lasting inhibition of faces over which semitransparent spatially nonpredictive cues were superimposed. The capacity for faces, in particular, to reinstate the inhibition that occurred during their initial presentation episode would seem to argue that cuing manipulations 
should be sensitive to whether the cues themselves are holistic facial configurations or not. Yet, on the basis of our Experiments 1 and 3, this is clearly not the case. We produced no evidence to suggest that face cues are more easily associated with an inhibitory state than are scrambled-face or nonface cues; all cue configurations produced equivalent IOR effects. The difference, however, is that in Tipper et al.'s (2003) study, each individual face stimulus served as a task-irrelevant background to which the spatial and/or object-based (e.g., Tipper, Driver, \& Weaver, 1991) inhibitory effects of IOR were tagged. This contrasts with our own experiments, for which the cues laid down the inhibitory tag. Thus, the IOR effects revealed in Experiments 1 and 3 were not for the cues themselves but for the locations at which the cues were presented. As such, our results do not challenge Tipper et al.'s (2003) claims for face-specific long-term inhibitory coding.

Equivalent IOR following face, scrambled-face, and nonface cues contradicts our initial prediction but is consistent with the presumed role that IOR plays in visual search. When an expressionless face-for instance, the one used in all three of our experiments - appears in the periphery, it would make evolutionary sense for ongoing behavior to be protected from repeated distraction from the location containing this irrelevant information. This is because a face with a neutral expression has no immediate survival implications; there would be no benefit from having attention linger at the cued location or from allowing this stimulus to alter ongoing behavior. Thus, a face cue with a neutral expression is functionally equivalent to any other kind of task-irrelevant environmental cue (e.g., a scrambled face or a nonface). As such, a face cue with a neutral expression results in the same withdrawal of (overt and/or covert) attention as does a scrambled-face or nonface cue and thereby results in the same inhibitory consequences. In contrast, when a face in the periphery communicates potential danger (e.g., threat or fear), it would make evolutionary sense for attention to remain focused on this stimulus and for IOR to thereby be overridden in favor of rapid responding to the information that arises from this potential source of danger. Indeed, this is exactly what happened in Fox et al.'s (2002) study: IOR effects tended not to occur following angry face cues. Although this was true of low anxious individuals in only one of two experiments in which IOR was tested (Experiment 2), it was true of high-anxious individuals in both experiments (Experiments 2 and 3 ). This suggests that the extent to which emotional expression may obviate the capacity for face cues to generate IOR depends, in part, on the observer's prepotent sensitivity to danger within the environment. Thus, interactions between the emotional expression of the face and the sensitivity of the observer may influence the generation of IOR, even if the mere presence of a face cue does not.

Evolutionary arguments suggest that once IOR is laid down, it may subserve effective visual search by preventing the repeated sampling of information from a location to which (overt and/or covert) attention has already been captured and withdrawn (e.g., Klein \& MacInnes, 1999). It may also subserve adaptive responding by slowing ongoing behavior enough to allow new stimuli in the environment to gain control over responding (Ivanoff \& Taylor, in press). To the extent that an evolved mechanism should be sensitive to stimuli that have potential biological relevance, it was expected that the magnitude of IOR would be affected by the configuration of the target stimuli in Experiments 2 and 3. The importance of social stimuli in our evolutionary history would seem to make it adaptive for face targets, even if expressionless, to suffer relatively less inhibition than do nonface targets. This evidently is not the case. IOR appears to be a "blind" mechanism that inhibits the spatial processing of subsequent stimuli, regardless of their visual characteristics. At least when the target response depends on localization (and hence, dorsal stream processing), there is no mechanism that allows preferential processing of face stimuli by the ventral stream to compete with and functionally override IOR. In other words, when emotionally neutral social stimuli are task irrelevant, ongoing behavior is as immune to the potentially distracting effects of these stimuli as to any other stimuli occurring in the broader visual environment.

In summary, the results of the present investigation reveal that the mere presence of face stimuli does not affect IOR. When there is no immediate threat or danger signaled by a cue stimulus, IOR is laid down, and ongoing behavior is subserved by preventing irrelevant stimuli from regaining control over attention/responding. Once laid down, IOR is a "blind" mechanism that slows responding to all emotionally neutral stimuli occurring at the affected location - at least, when location is used as the basis of responding. We are currently investigating whether IOR continues to be a "blind" mechanism when ventral stream processing is required in order to perform the target task.

\section{REFERENCES}

Cohen, J., MacWhinney, B., Flatt, M., \& Provost, J. (1993). PsyScope: An interactive graphic system for designing and controlling experiments in the psychology laboratory using Macintosh computers. Behavior Research Methods, Instruments, \& Computers, 25, 257-271.

DAnZIGER, S., \& Kingstone, A. (1999). Unmasking the inhibition of return phenomenon. Perception \& Psychophysics, 61, 1024-1037.

Dorris, M. C., Klein, R. M., Everling, S., \& Munoz, D. P. (2002). Contribution of the primate superior colliculus to inhibition of return. Journal of Cognitive Neuroscience, 14, 1256-1263.

Eastwood, J. D., Smilek, D., \& Merikle, P. M. (2001). Differential attentional guidance by unattended faces expressing positive and negative emotion. Perception \& Psychophysics, 63, 1004-1013.

Fox, E., Russo, R., \& Dutton, K. (2002). Attentional bias for threat: Evidence for delayed disengagement from emotional faces. Cognition \& Emotion, 16, 355-379.

Friesen, C. K., \& Kingstone, A. (1998). The eyes have it! Reflexive orienting is triggered by nonpredictive gaze. Psychonomic Bulletin \& Review, 5, 490-495.

Gallup, G. G., JR., Nash, R. F., \& Ellison, A. L., JR. (1971). Tonic immobility as a reaction to predation: Artificial eyes as a fear stimulus for chickens. Psychonomic Science, 23, 79-80.

Hietanen, J. K., \& Leppänen, J. M. (2003). Does facial expression affect attention orienting by gaze direction cues? Journal of Experimental Psychology: Human Perception \& Performance, 29, 1228-1243.

IVANOFF, J., \& TAYLOR, T. L. (in press). Inhibition of return promotes stop signal inhibition by delaying responses. Visual Cognition. 
Kanwisher, N., McDermott, J., \& Chun, M. M. (1997). The fusiform face area: A module in human extrastriate cortex specialized for face perception. Journal of Neuroscience, 17, 4302-4311.

Kingstone, A., \& Pratt, J. (1999). Inhibition of return is composed of attentional and oculomotor processes. Perception \& Psychophysics, 61, 1046-1054.

KLEIN, R. M. (1988). Inhibitory tagging system facilitates visual search. Nature, 334, 430-431.

KLEIN, R. M., \& MACInNES, W. J. (1999). Inhibition of return is a foraging facilitator in visual search. Psychological Science, 10, 346-352.

KleIN, R. M., \& TAYLOR, T. L. (1994). Categories of cognitive inhibition, with reference to attention. In D. Dagenbach \& T. H. Carr (Eds.), Inhibitory processes in attention, memory, and language (pp. 113150). San Diego: Academic Press.

Öhman, A., Lundevist, D., \& Esteves, F. (2001). The face in the crowd revisited: A threat advantage with schematic stimuli. Journal of Personality \& Social Psychology, 80, 381-396.

PosNer, M. I., \& CoHEN, Y. (1984). Components of visual orienting. In H. Bouma and D. G. Bouwhuis (Eds.), Attention and performance X: Control of language processes (pp. 531-556). Hillsdale, NJ: Erlbaum.

Posner, M. I., Rafal, R. D., Choate, L. S., \& Vaughan, J. (1985). Inhibition of return: Neural basis and function. Cognitive Neuropsychology, 2, 211-228

SAPIr, A., Soroker, N., Berger, A., \& HeniK, A. (1999). Inhibition of return in spatial attention: Direct evidence for collicular generation. Nature Neuroscience, 2, 1053-1054.

TAYLOR, T. L., \& Klein, R. M. (1998). On the causes and effects of inhibition of return. Psychonomic Bulletin \& Review, 5, 625-643.

TAYLOR, T. L., \& KLEIN, R. M. (2000). Visual and motor effects in inhibition of return. Journal of Experimental Psychology: Human Perception \& Performance, 26, 1639-1656.

TipPER, S. P., Driver, J., \& WeAVER, B. (1991). Object-centered inhibition of return of visual attention. Quarterly Journal of Experimental Psychology, 43A, 289-298.
Tipper, S. P., Grison, S., \& Kessler, K. (2003). Long-term inhibition of return of attention. Psychological Science, 14, 19-25.

Tong, F., Nakayama, K., Moscovitch, M., Weinrib, O., \& KANWISHER, N. (2000). Response properties of the human fusiform face area. Cognitive Neuropsychology, 17, 257-279.

\section{NOTE}

1. Fixation was purposely not controlled in the present investigation As is indicated, an adaptive mechanism is most likely to operate in the context of naturalistic viewing - that is, without constraints on eye and head movements. Indeed, as compared with more natural viewing conditions, the very nature of IOR is affected by explicit eye movement restraint, appearing to be a motor-based effect under restraint and a perceptual and/or attentional effect without restraint (e.g., Taylor \& Klein, $2000)$. Although the brief $(200-\mathrm{msec})$ cue and target presentations and relatively small visual field expanse $\left(5^{\circ}\right.$ from center to the outside edge of the peripheral stimulus boxes) likely minimized head and eye movements in the present experiment, it is nevertheless possible that overt orienting occurred to at least some of the cues and/or targets. As such, eye movements may have effectively altered the processing hemisphere, rendering the examination of left/right field effects meaningless. Because the key question was about the relative ability of facial configurations of cue and target stimuli to affect IOR, the inability to examine visual field effects does not have theoretical significance for the present investigation. For our purposes, it was more desirable to simulate natural viewing and obtain a "true" measure of IOR effects as a function of stimulus configuration than to alter the nature of IOR for the sake of being able to measure field effects.

(Manuscript received April 28, 2004;

revision accepted for publication February 25,2005 .) 